\title{
The Future of Pharmacist's Prescribing in Developing Countries
}

\author{
Asim Ahmed Elnour* \\ Department of Pharmacology, College of Medicine, UAE University, United Arab Emirates
}

Received: January 08, 2014, Accepted: January 10, 2014, Published: January 17, 2014

*Corresponding author: Asim Ahmed Elnour, Department of Pharmacology, College of Medicine, UAE University, United Arab Emirates, Tel: + 97150 6734096; E-mail: assahura1962@gmail.com

\section{Editorial}

The growing concern of medication errors and drug budget constrains are crucial to healthcare business. The use of computerized physician orders entry has placed enormous stress on the physicians in terms of, the time spend in prescribing pharmaceuticals, to selection of appropriate dosing and alerts of interactions. All these factors taken together may affect the physician's main objective of focusing on diagnosing the patient and placing laboratory tests and documenting patient clinical details.

Although the use of e-prescribing provides many advantages, nevertheless; it takes more time and induces more stress on physicians. This time in turn could be utilized for more elaborate clinical evaluation of patient's medical condition, quality of care and appropriate clinical decision making.

The process of prescribing is divided between the treating physician and the pharmacy where the patient receives his medications. Shortening this process by allowing the pharmacist to select the most appropriate pharmaceuticals for each particular patient may provide great opportunities for pharmacists to develop suitable pharmaceutical care plan, monitor and follow up their own prescribed medications and communicate and consult physicians for more confirmations.
The pharmacy colleges need to incorporate prescription pharmacy/dispensing pharmacy, at the core curriculum. The pharmacists associations worldwide can contribute to this era by launching workshops, conferences and targeting global concerns on increasing expenditure of use, misuse and overuse of pharmaceuticals.

There is a substantial need for research to evaluate the benefits of pharmacist's prescribing and assess its value with respect to drug budgets, quality of care and clinical health outcomes. More reliable research is needed for targeting specific patients groups, chronic diseases (diabetes, hypertension, asthma, ulcer etc...) and most common safely prescribed medications. Randomized clinical trials are of paramount importance to reveal the real benefits of 'prescription by a pharmacist' versus 'prescription by a physician'. We need to support the pharmacist prescribing experience by compelling evidence and setting up policies to govern this new trend.

Looking at the future, the pharmacist should take over and complete the process of 'prescribing and dispensing' by getting actively involved in every step of developing, manufacturing, storing, procuring and dispensing the pharmaceuticals. 